\title{
In-Plane Plasmonic Antenna Arrays with Surface Nanogaps for Giant Fluorescence Enhancement
}

\author{
Valentin Flauraud, ${ }^{\dagger}$ Raju Regmi, ${ }^{\ddagger}, \S$ Pamina M. Winkler, ${ }^{\S}$ Duncan T. L. Alexander, ${ }^{\prime \prime}$ Hervé Rigneault, ${ }^{\ddagger}$

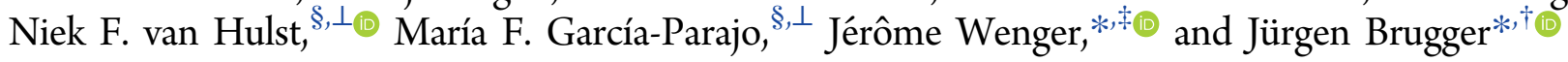 \\ ${ }^{\dagger}$ Microsystems Laboratory, Institute of Microengineering, Ecole Polytechnique Fédérale de Lausanne, 1015 Lausanne, Switzerland \\ ${ }^{\ddagger}$ Aix Marseille Univ, CNRS, Centrale Marseille, Institut Fresnel, F-13013 Marseille, France \\ ${ }^{\S}$ ICFO-Institut de Ciencies Fotoniques, The Barcelona Institute of Science and Technology, 08860 Castelldefels (Barcelona), Spain \\ "Interdisciplinary Center for Electron Microscopy (CIME) Ecole Polytechnique Fédérale de Lausanne, 1015 Lausanne, Switzerland \\ ${ }^{\perp}$ ICREA, Pg. Lluís Companys 23, 08010 Barcelona, Spain
}

Supporting Information

ABSTRACT: Optical nanoantennas have a great potential for enhancing light-matter interactions at the nanometer scale, yet fabrication accuracy and lack of scalability currently limit ultimate antenna performance and applications. In most designs, the region of maximum field localization and enhancement (i.e., hotspot) is not readily accessible to the sample because it is buried into the nanostructure. Moreover, current large-scale fabrication techniques lack reproducible geometrical control below $20 \mathrm{~nm}$. Here, we describe a new nanofabrication technique that applies planarization, etch back, and template stripping to expose the excitation hotspot at the surface, providing a major improvement over conventional electron beam lithography methods. We present large flat surface arrays of in-plane nanoantennas, featuring gaps as small as 10 $\mathrm{nm}$ with sharp edges, excellent reproducibility and full surface accessibility of the hotspot confined region. The novel fabrication approach drastically improves the optical performance of plasmonic nanoantennas to yield giant fluorescence enhancement factors up to $10^{4}-10^{5}$ times, together with nanoscale detection volumes in the $20 \mathrm{zL}$ range. The method is fully scalable and adaptable to a wide range of antenna designs. We foresee broad applications by the use of these in-plane antenna geometries ranging from large-scale ultrasensitive sensor chips to microfluidics and live cell membrane investigations.

KEYWORDS: Optical nanoantennas, template stripping, electron beam lithography, fluorescence enhancement, plasmonics

ptical nanoantennas take advantage of the plasmonic

response of noble metals to strongly confine light energy into nanoscale dimensions and breach the classical diffraction limit. $^{1-3}$ This confinement leads to a drastic enhancement of the interactions between a single quantum emitter and the light field, ${ }^{4-7}$ enabling large fluorescence gains above a thousand fold, ${ }^{8-13}$ ultrafast picosecond emission, ${ }^{14-16}$ and photobleaching reduction. ${ }^{17,18}$ As such, optical antennas hold great interest for ultrasensitive biosensing, especially for the detection of single molecules at biologically relevant micromolar concentrations. $^{19-21}$

Biosensing applications of nanoantennas require the largescale availability of narrow accessible gaps. Not only should nanogaps with sub-20 $\mathrm{nm}$ dimensions be reproducibly fabricated but also the gap region (plasmonic hotspot) must remain accessible to probe the target molecules. Despite impressive recent progress using electron beam, ${ }^{22}$ focused ion beam, ${ }^{23}$ or stencil lithographies ${ }^{24-26}$ or alternatively with bottom-up self-assembly techniques, ${ }^{6,7,9,13,16,27-30}$ the challenges of reliable narrow gap fabrication and hotspot accessibility remain major hurdles limiting the impact and performance of optical nanoantennas. For instance, when aiming for the fabrication of aperture antennas, electron beam lithography (EBL) using a positive-tone resist requires metal dry etching, which produces high line-edge roughness that are not suited for the definition of reliable and high aspect ratio nanogaps. Alternatively, patterning openings in metal films relying on EBL and negative-tone resist demands a lift-off approach. This is an efficient approach when lifting a full metal film for fabricating single particles but can be arduous when removing small isolated clusters of metal to clear apertures.

Here, we report on a novel nanofabrication technique based on EBL followed by planarization, etch back, and template stripping. The process provides large flat arrays of in-plane nanoantennas featuring $10 \mathrm{~nm}$ gaps with sharp edges and full accessibility of the localized hotspot illumination provided by

Received: November 30, 2016

Revised: February 6, 2017

Published: February 9, 2017 

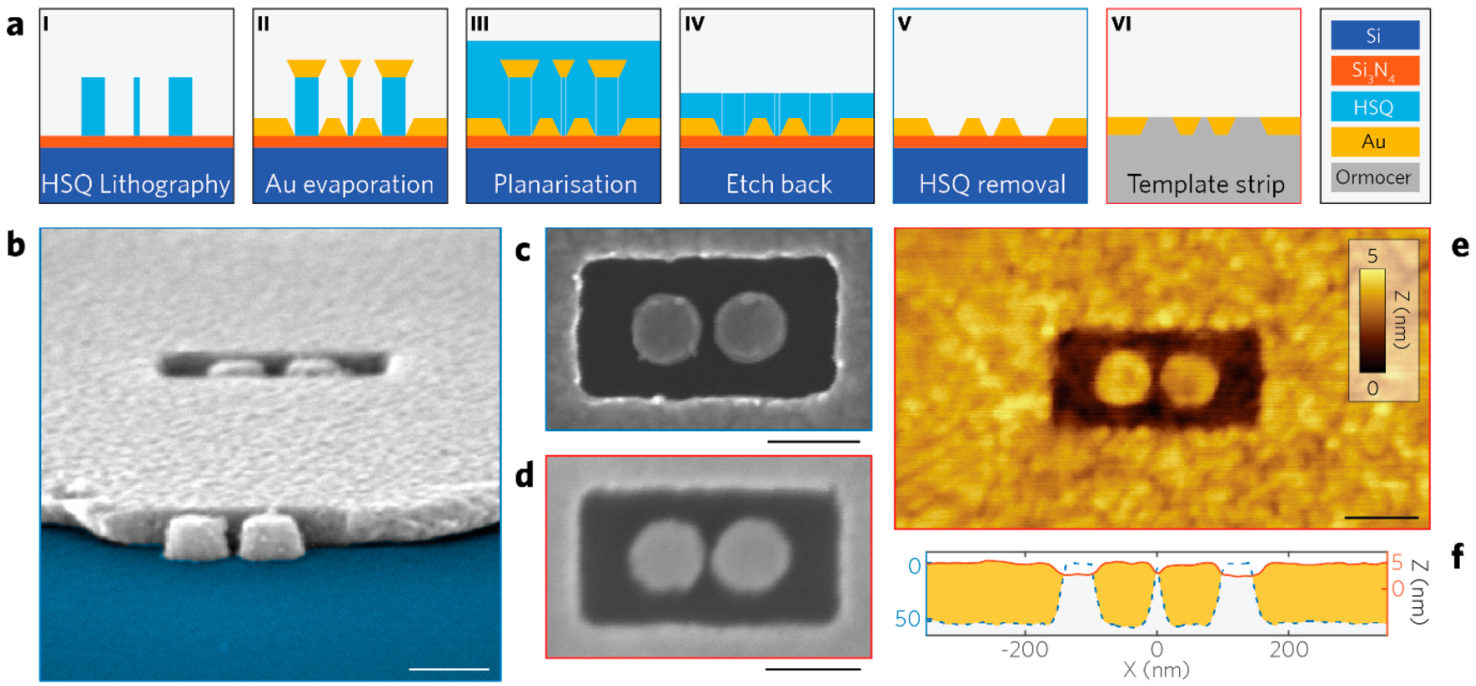

Figure 1. Fabrication of planar antenna arrays. (a) The antenna fabrication process flow is performed on a silicon nitride on silicon thin-film. The HSQ resist is patterned by electron-beam lithography (I) followed by gold evaporation (II), flowable oxide is spun for planarization (III) followed by etch back by Ar ion beam etching (IV), wet etching of the remaining HSQ (V) and final template stripping by UV curable adhesive (VI). (b) Tilted SEM view of an opened antenna-in-box before template stripping. The smallest part of the gap, here $\sim 12 \mathrm{~nm}$, lies at the surface level. A similar structure is imaged from the top before (c) and after (d) template striping. Dimensions are preserved and the space surrounding the antenna surrounding is filled by the UV curable polymer as seen in the AFM image (e) showing less than $5 \mathrm{~nm}$ residual topography. Panel (f) shows two AFM profiles averaged over 20 line scans before (dashed blue) and after (red) template stripping of the $50 \mathrm{~nm}$ thick gold structure. Scale bars are $100 \mathrm{~nm}$.

the antenna gap. These features enable single molecule fluorescence enhancement factors of $10^{4}-10^{5}$ and detection volumes in the $20 \mathrm{zL}$ range, outperforming previous plasmonic realizations. Because the method achieves excellent geometric control in the nanometer range over large areas, is fully adaptable to different antenna designs, and provides direct access to the enhanced field at the gap region, we foresee that this fabrication approach will significantly improve the effectiveness of plasmonic antennas for multiple applications, including ultrasensitive biosensing and live cell research.

The antenna design is based on the "antenna-in-box" platform featuring a nanogap dimer antenna inside a nanobox. ${ }^{10,31}$ This design is especially tailored for optimal enhanced single molecule analysis in solutions at high concentrations. It combines a central nanogap antenna between two $80 \mathrm{~nm}$ gold half-spheres to create the hotspot used for fluorescence enhancement, and a cladding $300 \times 140 \mathrm{~nm}^{2}$ box, to screen the background by preventing direct excitation of molecules diffusing away from the nanoantenna gap.

Figure 1a summarizes the different steps of our fabrication process. First, we use EBL on negative tone hydrogensilsesquioxane (HSQ) resist (step I Figure 1a). HSQ features a high-patterning resolution below $10 \mathrm{~nm},{ }^{32}$ as well as a highpostprocessing stability due to its inert inorganic $\mathrm{SiO}_{x}$ nature. After EBL patterning, a $50 \mathrm{~nm}$ thick gold film is deposited by electron beam evaporation at low temperature (step II) to reduce the gold grain size by approximately a factor of 2 as compared to room temperature evaporation (Figure S1). Then flowable oxide (HSQ) is spun to planarize the overall structure (step III) and so allow for a subsequent etch back (step IV) that selectively removes the sacrificial top metal layer in order to clear out the aperture geometry (Figure S2). This process is uniformly carried out at $100 \mathrm{~mm}$ wafer-scale and reliably results in the opening of all antennas at once after wet etching the HSQ (Step V). We point out that doing conventional lift-off without this etch back step is ineffective to remove the top metal sacrificial layer due to hydrophobic interactions (Figure S3).

Because of metal diffusion during the evaporation, the gold sidewalls bear a tapering angle. Therefore, the narrowest gap region lies at the bottom of the antenna close to the substrate interface, as shown in Figure $1 \mathrm{~b}$ and Figure S4. This hotspot position is impractical for biosensing and fluorescence enhancement applications, where the narrowest gap position should be on the top surface of the antenna to maximize the contact with the probe solution. We thus implement a template stripping approach (step VI Figure 1a) ${ }^{33,34}$ whereby the gold structures are transferred and flipped over onto a microscope coverslip to facilitate access to the narrowest and brightest region of the nanogap. Figure $1 c, d$ shows a comparison before and after template stripping, the gap size apparent on the top surface seems slightly reduced after stripping, mostly due to charging during imaging. Additionally, only the narrowest gap region emerges on a flat top surface (Figure 1e,f) enabling maximum fluorescence enhancement in a minimal near-field probe volume. Our fabrication method is fully general, allowing for the design of arbitrary planar geometries (Figure S5). It is conveniently performed on conductive silicon substrates so that the final structures may be subsequently transferred to arbitrary substrates such as microscope coverslips, avoiding the need for a supplementary adhesion layer that can damp the plasmonic performance. ${ }^{35}$ Additionally, the last template stripping step may be performed just before the final measurements, so the antenna hot spot is protected from surface contaminants during storage.

Transmission electron microscopy (TEM) is used to accurately quantify the dispersion in the antenna dimensions and the reproducibility of the fabrication method. The same production process, excluding template stripping, is carried out on a $30 \mathrm{~nm}$ thick freestanding silicon nitride membranes for accurate TEM metrology on prototypical arrays of nanoantennas (Figure 2a). Gap sizes from 10 to $45 \mathrm{~nm}$ are 


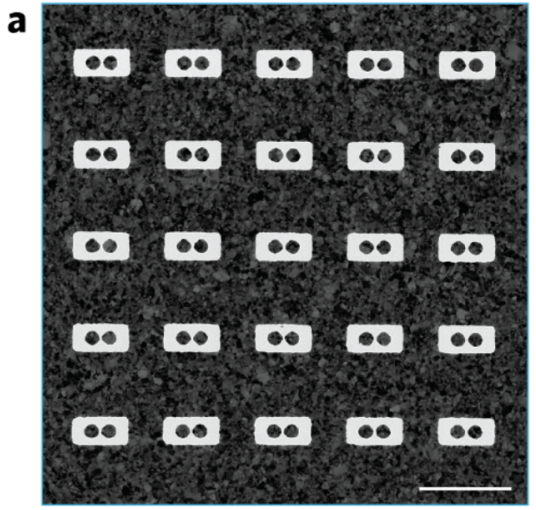

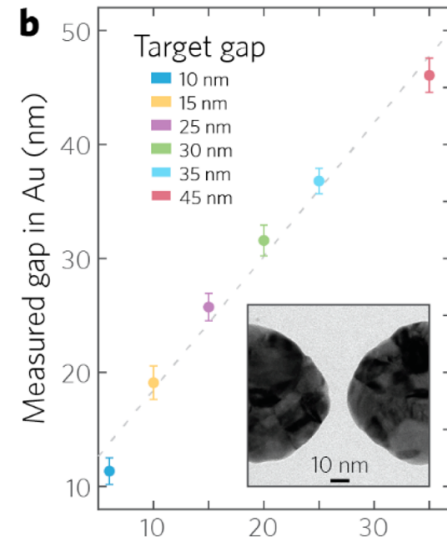

Gap width in resist $(\mathrm{nm})$

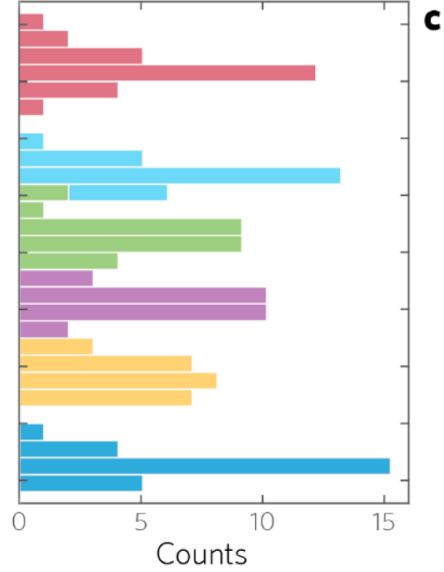

Figure 2. TEM metrology. (a) TEM image of a $5 \times 5$ antenna array with a $10 \mathrm{~nm}$ nominal gap width used for metrology purposes with no template stripping performed. The scale bar is $500 \mathrm{~nm}$. The rectangular apertures have $300 \mathrm{~nm}$ by $140 \mathrm{~nm}$ dimensions with each nanosphere being $80 \mathrm{~nm}$ in diameter. (b) Measured gap width of the Au dimers as a function of HSQ structure design width. Average gap width and associated standard deviation error bars are displayed for 6 sets of 25 antennas each. (c) Corresponding distribution histogram with 1.5 nm bin width. The gap size variations are due to the finite grain size in the Au film.
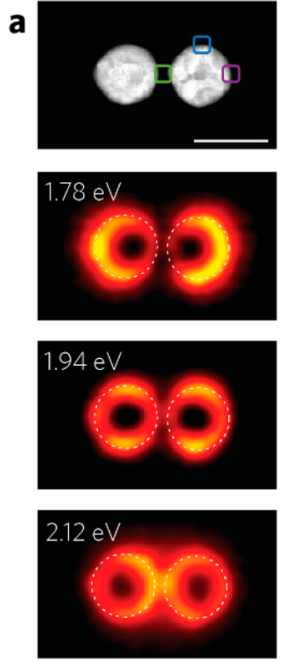

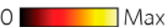
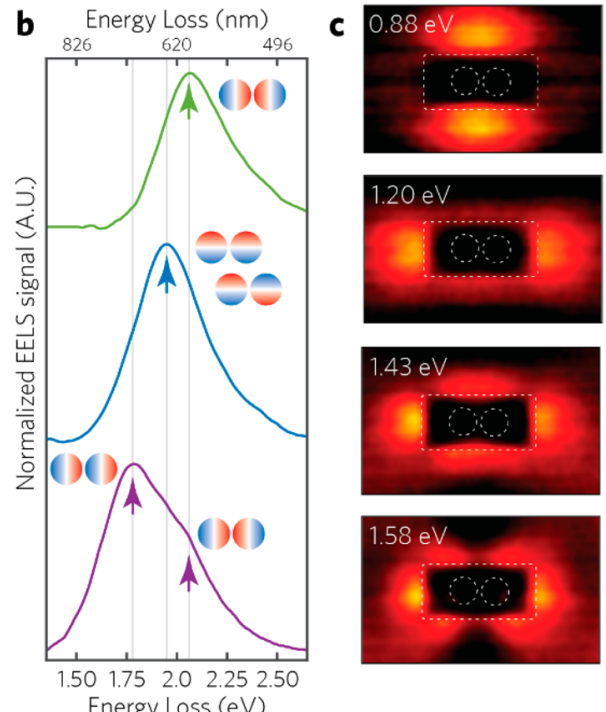

Energy Loss (eV)
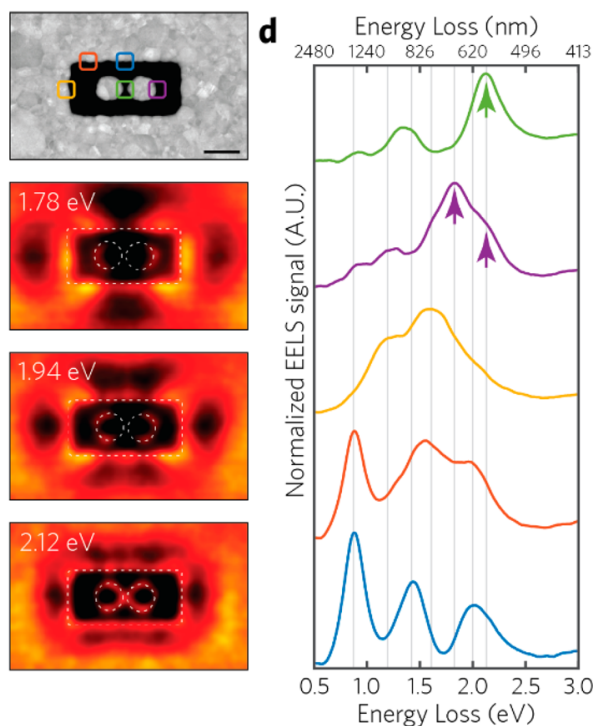

Figure 3. EELS analysis. (a) EELS maps at energy slices corresponding to the intensity maxima identified by extracting various spectra (b) at different impact parameters highlighted in the high-angle annular dark-field image, for an isolated dimer without surrounding box. The bonding and antibonding longitudinal dipoles are spatially and spectrally resolved at 1.78 and $2.12 \mathrm{eV}$, respectively. The bonding and antibonding transverse dipoles are revealed at $1.94 \mathrm{eV}$ with a characteristic EELS signal distribution along the sides of the dimer. In the case of the antenna-in-box (c,d), low energy modes at 0.88 and $1.2 \mathrm{eV}$ correspond to the fundamental dipole excited along the long or short axis of the rectangular box aperture, respectively. Multiple higher order harmonics are spatially and spectrally resolved from $1.43 \mathrm{eV}$ and higher. The mode excited at $1.78 \mathrm{eV}$ corresponds to the longitudinal bonding dipole of the dimer. A second mode, well identified in the green curve, corresponds to the expectedly higher energy $(2.12 \mathrm{eV})$ antibonding longitudinal dipole. The EELS colormap is scaled to the data range of each map and scale bars are $100 \mathrm{~nm}$.

fabricated with narrow dispersions (Figure 2b,c). Although HSQ is patterned at dimensions in the range of $5 \mathrm{~nm}$ for the nanogap region, the effective gap size of the gold dimer appears systematically larger. As already mentioned, this effect is due to a combination of metal diffusion and aperture clogging during evaporation, as well as metal wetting and diffusion onto the substrate. It should additionally be noted that the low feature density results in negligible contribution from proximity effects in EBL, and that the lithographic resolution is equivalent on bulk and membrane substrates, as it is inherently limited by forward scattering in both cases.

We then used scanning transmission electron energy-loss spectroscopy (STEM-EELS) to characterize the electro- magnetic properties of the antennas. The EELS response of the antenna-in-box can be quite complex because of the influence of the rectangular aperture surrounding the dimer antenna. Therefore, we first consider the simpler case of a single gold dimer without the surrounding metal layer (Figure 3a,b). Several modes are identified: at $2.12 \mathrm{eV}$ the longitudinal antibonding mode is efficiently excited by the electron beam, ${ }^{36}$ although presenting a vanishing net dipole moment, therefore being optically dark and not accessible for far field excitation. The peak at $1.94 \mathrm{eV}$ is assigned to both the bonding and antibonding transverse modes that present limited energy splitting as the transverse dipoles in each monomer are weakly coupled. Finally, the lowest energy mode $(1.78 \mathrm{eV})$ is the 

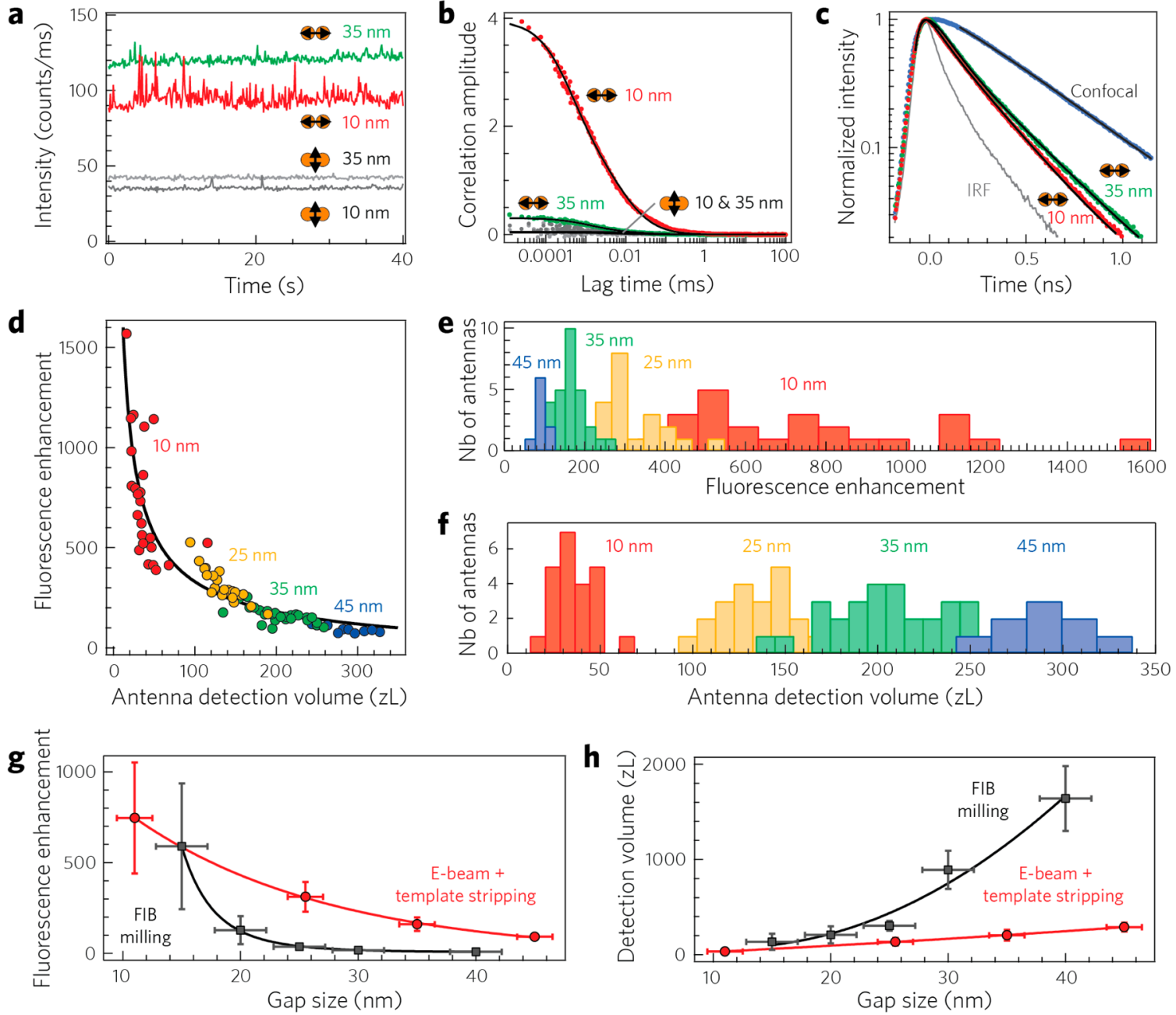

Figure 4. Nanoantennas enhance the fluorescence detection of Alexa Fluor 647 molecules in solution. (a) Fluorescence time traces and (b) corresponding FCS correlation functions (dots, raw data; lines, numerical fits) for nanoantennas with 10 and $35 \mathrm{~nm}$ gap sizes with the excitation polarization set parallel or perpendicular to the antenna's main axis. The experimental conditions correspond to $26 \mu \mathrm{M}$ of Alexa Fluor 647 with 200 $\mathrm{mM}$ of methyl viologen as chemical quencher under $2.3 \mathrm{~kW} / \mathrm{cm}^{2}$ excitation intensity at $633 \mathrm{~nm}$ wavelength. (c) Normalized time-resolved decay traces show Alexa Fluor 647 fluorescence lifetime reduction as the gap size is reduced. Black lines are numerical fits convoluted by the instrument response function (IRF). (d) Scatter plot of the fluorescence enhancement versus the nanoantenna's detection volume as deduced from FCS analysis on 83 different nanoantennas. The black line fit follows a power law dependence with a fixed $-2 / 3$ exponent. (e) Distribution of fluorescence enhancement factors deduced from the data in (d) for different gap sizes. (f) Distribution of the nanoantenna detection volume. (g,h) Average values of fluorescence enhancement $(\mathrm{g})$ and detection volume $(\mathrm{h})$ as a function of the gap size. The data for FIB milling is taken from ref 10 with similar conditions for the FCS experiments. Error bars correspond to one standard deviation.

optically active bonding longitudinal mode that we exploit to optically drive the gap enhancement. The lack of EELS signal in the gap region, originating from the cylindrical symmetry of the field produced by the electron beam, does not directly reveal the gap hotspot but further confirms the strong bonding nature of this mode. ${ }^{36}$ In the complete antenna-in-box configuration (Figure $3 \mathrm{c}, \mathrm{d}$ ), spectra and maps reveal a similar hybridization scheme for the dimer. The longitudinal bonding mode is again observed at $1.78 \mathrm{eV}$, in good qualitative agreement with the measured dark-field scattering spectrum (Figure S6a,b). Lastly, several low energy modes for the antenna-in-box are excited below $1.78 \mathrm{eV}$. These modes are not present for the isolated dimer (Figure 3a,b); we therefore relate them to the different multipoles of the surrounding box aperture. These aperture modes all occur at long wavelengths, do not overlap spectrally with the longitudinal bonding dimer mode and therefore have a minimal influence on the antenna's performance for fluorescence enhancement.
Fluorescence experiments assess the optical performance of the nanoantennas, and quantify the fluorescence enhancement together with the antenna's near-field volume. The experiments are performed by covering the sample with a solution containing Alexa Fluor 647 or crystal violet fluorescent molecules at micromolar concentrations. The absorption and emission spectra of both dyes feature a strong overlap with the antenna's resonance (Figure S6c,d). The fluorescence signal is analyzed with fluorescence correlation spectroscopy (FCS) and fluorescence burst analysis. FCS determines the average number of detected molecules from which we deduce the fluorescence brightness per emitter and the detection volume (see Methods for details). In order to maximize the fluorescence enhancement brought by the nanoantenna, we use low quantum yield emitters: $200 \mathrm{mM}$ of methyl viologen is added to the solution to quench the Alexa 647 quantum yield to $8 \%$, and the quantum yield of crystal violet is around $2 \%$. $^{10,37-39}$ 
Figure $4 \mathrm{a}, \mathrm{b}$ displays the raw fluorescence intensity time traces and corresponding correlation curves with excitation polarization parallel and perpendicular to the antenna dimer axis for two different gap sizes of 10 and $35 \mathrm{~nm}$. Larger fluorescence fluctuations and higher correlation amplitudes are clearly observed when the incident electric field is parallel to the antenna axis and when the gap size is reduced. This directly evidence the presence of an electromagnetic hotspot in the antenna gap region. All experiments are performed at $26 \mu \mathrm{M}$ concentration of Alexa Fluor 647, corresponding to 7630 molecules in the $0.5 \mathrm{fL}$ confocal detection volume (Figure S7). The FCS correlation amplitude scales as the inverse of the number of fluorescent molecules, so in the confocal reference without the nanoantenna, the FCS amplitude is very low at 1 / $7630=1.3 \times 10^{-4}$. In contrast, correlation amplitudes of 3.9 are detected with the nanoantenna of $10 \mathrm{~nm}$ gap size and correspond to an average number of 0.26 molecules. The antenna detection volume can thus be quantified to $17 \mathrm{zL}(1 \mathrm{zL}$ $=10^{-21} \mathrm{~L}=1000 \mathrm{~nm}^{3}$ ) using the known $26 \mu \mathrm{M}$ fluorophore concentration. This volume is the smallest reported for FCS applications on nanoantennas ${ }^{8-11,27,36}$ and corresponds to a value 30000 times lower than the diffraction-limited confocal volume. The reduction of the detection volume is confirmed by the shortening of the diffusion time from $64 \mu \mathrm{s}$ in the diffraction-limited confocal volume to $0.9 \mu \mathrm{s}$ in the nanoantenna. For a molecule undergoing Brownian diffusion, the root-mean-square of the displacement scales as $(2 D t)^{1 / 2}$, where $D$ is the diffusion coefficient and $t$ the elapsed time. Using this formula with $D=300 \mu \mathrm{m}^{2} / \mathrm{s}$ for Alexa Fluor 647 at $21^{\circ} \mathrm{C}^{40}$ and $t=0.9 \mu \mathrm{s}$, we get a typical size of $23 \mathrm{~nm}$, which corresponds to a $50 \mathrm{zL}$ volume. While this number confirms the detection volume estimated from the FCS correlation amplitude, this approach cannot be used for an accurate measurement due to the complex $3 \mathrm{D}$ form of the antenna hot spot volume and the presence of the interface influencing the Brownian diffusion.

For some experiments, ${ }^{10,29}$ the background fluorescence from molecules diffusing away from the hotspot can overwhelm the signal from the hot spot and complicate the FCS analysis. Here, we find that the signal from the hotspot always largely dominates the background, so that the previously used corrections are no longer needed. The number of detected molecules is simply the inverse of the correlation amplitude at zero lag time, and the fluorescence brightness per emitter can be computed by normalizing the average fluorescence intensity by this number of detected molecules. For the antenna with 10 $\mathrm{nm}$ gap size, we find a brightness of 370 counts $/ \mathrm{ms}$. This value is 1600 times higher than the 0.24 counts $/ \mathrm{ms}$ found for the dye in the confocal reference setup (Figure S7) and clearly demonstrates the occurrence of large fluorescence enhancement in the nanogap. Simultaneously, the fluorescence lifetime is significantly reduced from $380 \pm 15$ ps in confocal illumination to $45 \pm 10 \mathrm{ps}$ in the $10 \mathrm{~nm}$ gap antenna (Figure $4 \mathrm{c}$ and Figure S8).

Several additional test experiments confirm the near-field origin of the FCS signal. First, all the relevant observables, nanoscale volume confinement, fluorescence enhancement, and lifetime reduction, disappear when the laser polarization is oriented perpendicular to the main antenna axis or when the gap size is increased. We have also performed control experiments on an antenna sample with an extra $8 \mathrm{~nm}$ thick silica layer deposited on top of the antennas to prevent the molecules from accessing the hotspot region. In that case, the FCS signal is lost (Figure S9) confirming the crucial role of the few nanometers region surrounding the antenna gap. A study of the excitation power dependence validates that no saturation, photobleaching or triplet blinking affect our data (Figure S10). We also checked that the residual background luminescence from the gold antenna (in the absence of fluorescent molecules) remains negligible and shows no temporal correlation (Figure S11).

To assess the statistical reproducibility of the antenna fabrication, we repeat the FCS experiments on a set of more than 80 different antennas and measure for each antenna its fluorescence enhancement and near-field detection volume. The scatter plot in Figure $4 \mathrm{~d}$ indicates a clear correlation between the fluorescence enhancement and the detection volume following an empirical power law with $-2 / 3$ exponent. This exponent value can be understood as the volume scales as the cube power of the typical near-field size, while the fluorescence enhancement is dominated by the gain in local excitation intensity which scales as the square power of the typical near-field size. For each value of the desired nominal gap size, the histograms of the fluorescence enhancement and detection volume illustrate the statistical dispersion of the data around the average (Figure 4e,f). This dispersion comes as natural consequence of the variability of the gap sizes as characterized by TEM in Figure 2b,c especially for the smallest gaps where a nanometer variation in the gap size can have a large influence on the antenna's performance and the measured enhancement factor.

Importantly, the performance of these new optical nanoantennas significantly outperforms the values achieved previously using focused ion beam lithography. ${ }^{10}$ Figure $4 \mathrm{~g}, \mathrm{~h}$ compares the average values of fluorescence enhancement and detection volume obtained for nanoantennas fabricated with conventional focused ion beam (FIB) milling or our technique. For a more detailed view, Figure S12 displays a scatter plot of the fluorescence enhancement versus the detection volume reduction. The nanoantennas were tested under similar conditions for FCS experiments to ensure a relevant benchmarking. As clearly demonstrated in Figures $4 \mathrm{~g}, \mathrm{~h}$ and S12, template-stripped E-beam lithography provides higher fluorescence enhancement factors in smaller detection volumes. The improvement over FIB milling is especially worthwhile for gap sizes above $25 \mathrm{~nm}$, where our technique can lead to a 10 times increase for the fluorescence enhancement factor together with a $5 \times$ reduction for the detection volume.

Emitters with low quantum yields allow reaching higher fluorescence enhancement factors, as the nanoantenna provides a larger benefit to increase the emission quantum yield. ${ }^{8,13}$ To probe this effect, we perform experiments on crystal violet (CV) molecules, which have $2 \%$ quantum yield. ${ }^{11,12}$ The CV solution is set to a concentration of $1 \mu \mathrm{M}$ in a water/glycerol 1:1 solution to slow down the diffusion of molecules crossing the antenna hotspot allowing for direct analysis of the fluorescence bursts for individual molecules. Using the detection volume estimated previously with FCS, the $1 \mu \mathrm{M}$ concentration ensures that on average less than 0.02 crystal violet molecules are present in the $10 \mathrm{~nm}$ gap region. This low number rules out the possibility that the estimated count rates per burst originate from more than one single molecule diffusing in the nanogap. Intense fluorescence bursts are clearly detected on the fluorescence time traces (Figure $5 a-c$ ) with their amplitude decreasing as the gap size is enlarged. This feature confirms that the fluorescence bursts stem from the antenna gap region. To measure the fluorescence enhancement, 


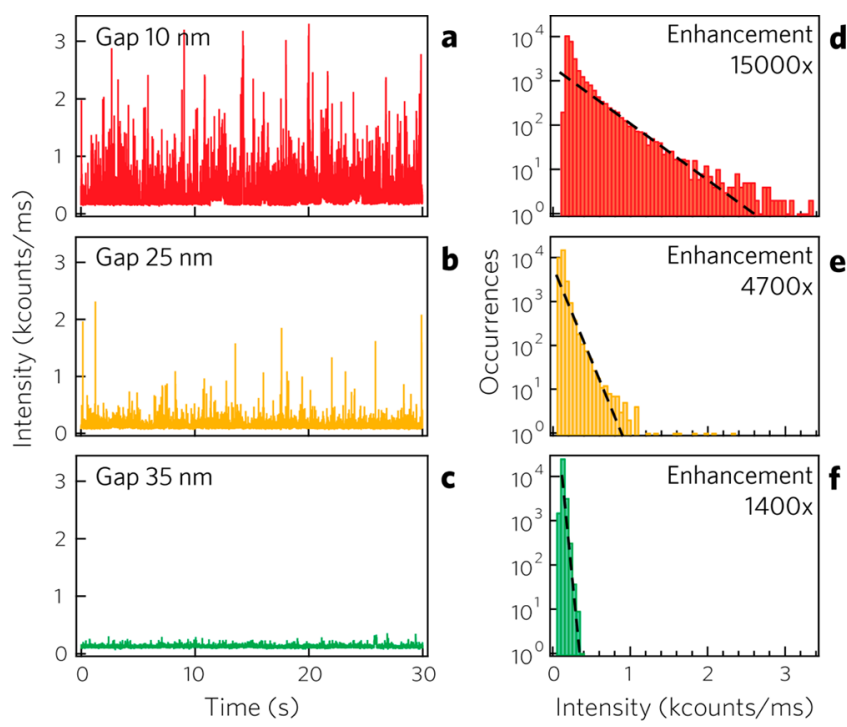

Figure 5. Fluorescence enhancement on crystal violet molecules measured with fluorescence bursts analysis. $(a-c)$ Fluorescence time traces recorded on nanoantennas with increasing gap sizes using $1 \mu \mathrm{M}$ of crystal violet in water/glycerol 1:1 solution with $2.3 \mathrm{~kW} / \mathrm{cm}^{2}$ excitation intensity at $633 \mathrm{~nm}$. The binning time is $1 \mathrm{~ms}$. (d-f) Photon count rate histograms deduced from the traces in $(a-c)$. The dashed lines are fits by exponentially decaying probability distributions.

we fit the photon count histograms in Figure $5 \mathrm{~d}-\mathrm{f}$ with exponentially decaying probability distributions and record the maximum peak amplitude in the fitted distribution. The reference peak fluorescence count per $\mathrm{CV}$ molecule is estimated at 0.18 counts $/ \mathrm{ms}$ at the same $2.30 \mathrm{~kW} / \mathrm{cm}^{2}$ excitation power (Figure S13), which is in agreement with values reported independently in refs 11 and 12. For the smallest $10 \mathrm{~nm}$ gap size, the maximum count is 2750 counts/ ms with a background of 100 counts/ms (set by the fluorescence from the $\mathrm{CV}$ molecules diffusing away from the hotspot region and the residual photoluminescence from the metal). This leads to an impressive fluorescence enhancement of 15000 times. The same procedure performed on Alexa Fluor 647 with $200 \mathrm{mM}$ methyl viologen (8\% quantum yield) indicates a fluorescence enhancement of $5300 \times$ for a $10 \mathrm{~nm}$ gap size (Figures S14 and S15). The relative change in the enhancement factors result from the differences of the quantum yields between $\mathrm{CV}$ and Alexa 647 measurements, thus confirming our measurements. Note that the enhancement factor for Alexa 647 estimated from the burst peak intensity is about 3 times higher than the one measured with FCS (Figure 4 ), as the burst analysis favors the best event when the emitter's position and orientation lead to the highest fluorescence intensity. To avoid the strong dependence of the fluorescence enhancement on the intrinsic quantum yield of the fluorescent reporter, the fluorescence enhancement figure of merit was defined as the product of the enhancement factor by the reference quantum yield of the emitter in homogeneous medium. ${ }^{13}$ For crystal violet and Alexa Fluor 647, the fluorescence enhancement figures of merit amount to 300 and 420 , respectively, and are the highest reported values to date. $^{8-13,16}$

The measured fluorescence enhancement factors come very close to the values predicted theoretically using the formula ${ }^{41}$

$$
\eta_{\mathrm{F}}=\frac{I_{\mathrm{exc}}^{*}}{I_{\mathrm{exc}}} \frac{\Gamma_{\mathrm{rad}}^{*}}{\Gamma_{\mathrm{rad}}} \frac{1}{1-\phi_{0}+\frac{\phi_{0}\left(\Gamma_{\mathrm{rad}}^{*}+\Gamma_{\text {loss }}^{*}\right)}{\Gamma_{\mathrm{rad}}}}
$$

This equation states that the fluorescence enhancement $\eta_{\mathrm{F}}$ is the product of the excitation intensity enhancement in the nanogap $I_{\mathrm{exc}}^{*} / I_{\mathrm{exc}}$ times the enhancement of the radiative decay rates $\Gamma_{\text {rad }}^{*} / \Gamma_{\text {rad }}$ and a third term that depends on the initial quantum yield $\phi_{0}$ of the fluorescent molecule and an additional decay rate $\Gamma_{\text {loss }}^{*}$ describing the nonradiative energy transfer to the antenna's material induced by ohmic losses. Here we neglect the collection efficiency improvement brought by the antenna (back focal plane imaging confirms this assumption $\left.^{10,42}\right)$. For the smallest $10 \mathrm{~nm}$ gap and a dipole emitter located in the gap center, the FDTD simulations estimate the different contributions to be $I_{\text {exc }}^{*} / I_{\text {exc }}=600, \Gamma_{\text {rad }}^{*} / \Gamma_{\text {rad }}=700$ and $\left(\Gamma_{\text {rad }}^{*}+\Gamma_{\text {loss }}^{*}\right) / \Gamma_{\text {rad }}=1100$ (Figure S16). These values predict fluorescence enhancement of 18000 times for crystal violet and 4700 times for Alexa 647, which come in excellent agreement with the experimental data.

In conclusion, we have described the combination of EBL followed by postprocessing and template stripping as a powerful and versatile method to fabricate nanoantennas with direct accessibility of the hotspot region, large-scale availability, and gap sizes as small as $10 \mathrm{~nm}$ with sharp edges. This design provides fluorescence enhancement factors up to 15000 times, together with nanoscale detection volumes in the range of 10 $\mathrm{zL}$. The present method can be applied to many other antenna designs while being fully scalable. Improvement on antenna fabrication opens up the possibility of fully exploiting the physical properties of plasmonic antennas for a whole range of applications, including biosensing and/or live cell research at the nanoscale.

Methods. Sample Fabrication. Silicon wafers $(100 \mathrm{~mm}$ diameter, prime grade) were cleaned following a standard RCA procedure prior to the low pressure chemical vapor deposition of $100 \mathrm{~nm}$ thick silicon nitride. Hydrogen silsesquioxane (HSQ) 4\% (Dow Corning) was spun at $1500 \mathrm{rpm}$ for $240 \mathrm{~s}$ yielding an approximately $100 \mathrm{~nm}$ thick coating. The samples were then exposed by electron beam lithography (VISTEC EBPG5000+, $100 \mathrm{kV}$ ) using a $1 \mathrm{~nm}$ grid and a $2 \mathrm{nA}$ beam (5 $\mathrm{nm}$ FWHM). Short range dose corrections were used to increase feature accuracy and reliably pattern the sub-10 nm features that define the narrowest gaps. After exposure, the samples were developed at room temperature in $25 \%$ tetramethylammonium hydroxide (TMAH) for $2 \mathrm{~min}$, rinsed in deionized water and isopropanol prior to drying in order to avoid capillary force-induced collapse of the narrowest features. A gold layer of $50 \mathrm{~nm}$ thickness was then evaporated by electron beam heating at a pressure of $8 \times 10^{-7} \mathrm{mBar}$ on static substrates ensuring normal incidence of the metal flux. The stage was cooled at $-50{ }^{\circ} \mathrm{C}$ throughout the evaporation (Huber unistat $705 \mathrm{w}$ ) to ensure small grain size allowing for high feature accuracy. In order to planarize the sample, flowable oxide (Dow Corning FOX-16) was then spun at $1000 \mathrm{rpm}$ for $240 \mathrm{~s}$ yielding a $1 \mu \mathrm{m}$ thick film with a residual topography above the structures of interest below $10 \mathrm{~nm}$. Broad argon ion beam milling (Veeco Nexus IBE350) performed at $-45^{\circ}$ sample tilt was then used to etch back the flowable oxide until the top gold caps were fully etched. End point detection was performed by monitoring the gold signal on a secondary ion mass spectrometer. A $30 \mathrm{~s}$ etch with hydrofluoric acid diluted 1:10 in deionized water was used to clear out the residual HSQ 
in the antenna apertures. The wafer was then cleaved into individual dies. For template stripping, microscope coverlips (30 $\mathrm{mm}$ diameter $150 \mu \mathrm{m}$ thickness) were cleaned in piranha solution and surface activated by oxygen plasma (Tepla Gigabtach 1000W, $500 \mathrm{SCCM} \mathrm{O}_{2}$ ) before being brought into contact with the gold substrates with a small drop of UV curable OrmoComp (microresist technology GMBH) and cross-linked under UV and light pressure (ESCO EUN-4200 $375 \mathrm{~nm}, 2.5 \mathrm{~mW} / \mathrm{cm} 2$ ) for $3 \mathrm{~min}$ followed by separation of the glass from the silicon with a razor blade. Additional samples were fabricated to perform high-resolution metrology and EELS characterization following a similar process without template stripping. The TEM membranes were fabricated at wafer-scale by using $30 \mathrm{~nm}$ LPCVD silicon nitride that was released in $100 \times 500 \mu \mathrm{m}^{2}$ windows from the backside by potassium hydroxide wet etching.

Metrology and Statistics. Sample imaging was performed by scanning electron microscopy (Zeiss Merlin) to measure the features before $(20 \mathrm{kV}, 360 \mathrm{pA}, 1 \mathrm{~mm}$ working distance and inlens detector) and after template stripping $(2 \mathrm{kV}, 80 \mathrm{pA}, 3 \mathrm{~mm}$ working distance, and in-lens detector). Topography was measured by atomic force microscopy (Bruker FastScan) in both ScanAsyst PeakForce Tapping and tapping mode. For high-resolution metrology statistics, imaging was performed both in TEM and STEM mode (FEI Talos) and processed with a custom matlab toolbox.

EELS Measurements. STEM-EELS maps were acquired using a FEI Titan Themis 60-300 equipped with a Wien-type monochromator and a Gatan GIF Quantum ERS spectrometer. A $300 \mathrm{keV}$ incident electron beam was used for all experiments, monochromated to give an energy spread of $\sim 110 \mathrm{meV}$ fwhm in the zero-loss peak of elastically scattered electrons and with beam currents of $\sim 240 \mathrm{pA}$. A $17 \mathrm{mrad}$ convergence semiangle of the probe and a 22 mrad collection semiangle on the spectrometer were used, with the probe having a mean diameter of $<1 \mathrm{~nm}$ for full width at tenth-maximum in incident intensity. Mapping was performed using the "ultrafast" spectrum imaging mode with typical dwell times of 0.20 to $0.26 \mathrm{~ms}$ per pixel and with the probe rastered in $X, Y$ step sizes of $0.5-0.6 \mathrm{~nm}$ for a total of $>10^{5}$ pixels per map. Each map was treated with the HQ Dark Correction plugin to reduce noise associated with dark current subtraction.

EELS Data Processing. The EELS data cubes were processed using Gatan Digital Micrograph and custom Matlab scripts for the removal of the background from the tails of the zero-loss peak (ZLP), extraction of point spectra and spatial EELS maps. The ZLP was first centered pixel by pixel using a GaussianLorentzian approximation. Following zero-loss alignment, each data cube was spectrally cropped to the region of interest including ZLP ( -2 to $4 \mathrm{eV})$, and artifacts from cosmic rays were removed. To account for the absorption and scattering inside the $\mathrm{Au}$ film, the data cubes were normalized by dividing each pixel-spectrum by the integrated zero-loss fit. Spectra presented in Figure 3 were integrated over a $30 \times 30$ pixel region of interest centered around the point overlaid on the STEM image, whereas EELS maps were typically integrated over a window of $0.06 \mathrm{eV}$ in energy range.

Fluorescence Experimental Setup. The experiments use an inverted confocal microscope with a Zeiss $40 \times, 1.2 \mathrm{NA}$ waterimmersion objective, and a three-axis piezoelectric stage allowing to select individual nanoantennas. The excitation for FCS and fluorescence burst experiments was provided by a linearly polarized $\mathrm{He}-\mathrm{Ne}$ laser at $633 \mathrm{~nm}$. For fluorescence lifetime measurements, the excitation source was a picosecond laser diode operating at $636 \mathrm{~nm}$ (Pico-Quant LDH-P-635). The fluorescence signal was collected in epi-detection mode through a dichroic mirror and a stack of two long-pass $650 \mathrm{~nm}$ filters to reject the backscattered laser light and maximize fluorescence collection. The detection was performed by two avalanche photodiodes (PicoQuant MPD-5CTC) after passing through a $30 \mu \mathrm{m}$ pinhole conjugated to the focus plane. The fluorescence time traces for burst analysis and lifetime histograms were recorded on a fast time-correlated single photon counting module in time-tagged time-resolved mode (PicoQuant PicoHarp 300). The concentration of fluorescent molecules was measured with extinction spectroscopy and confirmed by confocal FCS experiments on a series of dilutions. In the photon count histograms, we determine the peak fluorescence intensity by the intercept of the fitted exponential decay with the $x$-axis at $10^{\circ}$. Events of lower probabilities within the $30 \mathrm{~s}$ experiment duration and events lying above this level (corresponding to the presence of two molecules within the hot spot) are discarded.

Fluorescence Correlation Spectroscopy. The temporal fluctuations of the fluorescence intensity $F(t)$ were analyzed with a hardware correlator (Flex02-12D/C correlator.com, Bridgewater $\mathrm{NJ}$ ) to compute the temporal correlation of the fluorescence signal $G(\tau)=\langle\delta F(t) \delta F(t+\tau)\rangle /\langle F(t)\rangle^{2}$, where $\delta F(t)=F(t)-\langle F(t)\rangle$ is the fluctuation of the fluorescence signal around the average value, $\tau$ is the delay (lag) time, and \langle $>$ indicates time averaging. While our earlier works on plasmonic antennas required a special data treatment to compensate for the background fluorescence from molecules diffusing away from the hotspot, ${ }^{10,29}$ we find here that the signal from the hotspot largely dominates the background with the new antenna design, so that the previously used correction is no longer needed. This further simplifies the FCS analysis so that the temporal correlation of the fluorescence intensity $F$ can be written as

$$
G(\tau)=\frac{1}{N^{*}} \frac{1}{\left(1+\frac{\tau}{\tau_{\mathrm{d}}}\right) \sqrt{1+\frac{s^{2} \tau}{\tau_{\mathrm{d}}}}}
$$

where $N^{*}$ is the number of molecules in the gap region, $\tau_{\mathrm{d}}$ is the mean residence time, and $s$ is the ratio of transversal to axial dimensions of the analysis volume. $N^{*}$ can be estimated by taking the inverse of the correlation amplitude near zero lag time. The fluorescence brightness per molecule is finally deduced as $\langle F\rangle / N^{*}$.

\section{ASSOCIATED CONTENT}

\section{S Supporting Information}

The Supporting Information is available free of charge on the ACS Publications website at DOI: 10.1021/acs.nanolett.6b04978.

Gold evaporation and surface roughness, etch back process, comparison with conventional lift-off, gold evaporation driven clogging, other geometry examples, overlap between antenna's resonance and fluorescence spectra, FCS reference data for Alexa Fluor 647, fluorescence lifetime reduction and LDOS enhancement, control experiment with extra $8 \mathrm{~nm}$ silica, powerdependence study, luminescence background, performance benchmarking versus focused ion beam milling, fluorescence reference data for crystal violet, fluorescence 
bursts analysis on Alexa Fluor 647 molecules, reference fluorescence bursts analysis for Alexa Fluor 647, numerical simulations (PDF)

\section{AUTHOR INFORMATION}

\section{Corresponding Authors}

*E-mail: jerome.wenger@Fresnel.fr.

*E-mail: juergen.brugger@epfl.ch.

\section{ORCID}

Valentin Flauraud: 0000-0002-1393-3198

Niek F. van Hulst: 0000-0003-4630-1776

Jérôme Wenger: 0000-0002-2145-5341

Jürgen Brugger: 0000-0002-7710-5930

\section{Notes}

The authors declare no competing financial interest.

\section{ACKNOWLEDGMENTS}

The authors thank the staff of the Center of Micro/ Nanotechnology (CMI) of EPFL for the valuable discussions and support. The research leading to these results has received funding from the European Commission's Seventh Framework Programme (FP7-ICT-2011-7) under grant agreements ERC StG 278242 (ExtendFRET), 288263 (NanoVista) and ERC Adv. Grant 670949 (LightNet), Spanish Ministry of Economy and Competitiveness ("Severo Ochoa" Programme for Centres of Excellence in R\&D (SEV-2015-0522)) and Fundació CELLEX (Barcelona). R.R. is supported by the Erasmus Mundus Doctorate Program Europhotonics (Grant 159224-12009-1-FR-ERA MUNDUS-EMJD).

\section{REFERENCES}

(1) Novotny, L.; van Hulst, N. Nat. Photonics 2011, 5 (2), 83-90.

(2) Biagioni, P.; Huang, J.-S.; Hecht, B. Rep. Prog. Phys. 2012, 75 (2), 024402.

(3) Giannini, V.; Fernández-Domínguez, A. I.; Heck, S. C.; Maier, S. A. Chem. Rev. 2011, 111 (6), 3888-3912.

(4) Anger, P.; Bharadwaj, P.; Novotny, L. Phys. Rev. Lett. 2006, 96 (11), 113002.

(5) Kühn, S.; Håkanson, U.; Rogobete, L.; Sandoghdar, V. Phys. Rev. Lett. 2006, 97 (1), 017402.

(6) Chikkaraddy, R.; de Nijs, B.; Benz, F.; Barrow, S. J.; Scherman, O. A.; Rosta, E.; Demetriadou, A.; Fox, P.; Hess, O.; Baumberg, J. J. Nature 2016, 535 (7610), 127-130.

(7) Benz, F.; Schmidt, M. K.; Dreismann, A.; Chikkaraddy, R.; Zhang, Y.; Demetriadou, A.; Carnegie, C.; Ohadi, H.; de Nijs, B.; Esteban, R.; Aizpurua, J.; Baumberg, J. J. Science 2016, 354 (6313), 726-729.

(8) Kinkhabwala, A.; Yu, Z.; Fan, S.; Avlasevich, Y.; Mullen, K.; Moerner, W. E. Nat. Photonics 2009, 3 (11), 654-657.

(9) Acuna, G. P.; Möller, F. M.; Holzmeister, P.; Beater, S.; Lalkens, B.; Tinnefeld, P. Science 2012, 338 (6106), 506.

(10) Punj, D.; Mivelle, M.; Moparthi, S. B.; van Zanten, T. S.; Rigneault, H.; van Hulst, N. F.; Garcia-Parajo, M. F.; Wenger, J. Nat. Nanotechnol. 2013, 8 (7), 512-516.

(11) Yuan, H.; Khatua, S.; Zijlstra, P.; Yorulmaz, M.; Orrit, M. Angew. Chem., Int. Ed. 2013, 52 (4), 1217-1221.

(12) Khatua, S.; Paulo, P. M. R.; Yuan, H.; Gupta, A.; Zijlstra, P.; Orrit, M. ACS Nano 2014, 8 (5), 4440-4449.

(13) Puchkova, A.; Vietz, C.; Pibiri, E.; Wünsch, B.; Sanz Paz, M.; Acuna, G. P.; Tinnefeld, P. Nano Lett. 2015, 15 (12), 8354-8359.

(14) Akselrod, G. M.; Argyropoulos, C.; Hoang, T. B.; Ciracì, C.; Fang, C.; Huang, J.; Smith, D. R.; Mikkelsen, M. H. Nat. Photonics 2014, 8 (11), 835-840.

(15) Hoang, T. B.; Akselrod, G. M.; Mikkelsen, M. H. Nano Lett. 2016, 16 (1), 270-275.
(16) Bidault, S.; Devilez, A.; Maillard, V.; Lermusiaux, L.; Guigner, J. M.; Bonod, N.; Wenger, J. ACS Nano 2016, 10 (4), 4806-4815.

(17) Pellegrotti, J. V.; Acuna, G. P.; Puchkova, A.; Holzmeister, P.; Gietl, A.; Lalkens, B.; Stefani, F. D.; Tinnefeld, P. Nano Lett. 2014, 14 (5), 2831-2836.

(18) Wientjes, E.; Renger, J.; Cogdell, R.; van Hulst, N. F. J. Phys. Chem. Lett. 2016, 7 (9), 1604-1609.

(19) Levene, M. J.; Korlach, J.; Turner, S. W.; Foquet, M.; Craighead, H. G.; Webb, W. W. Science 2003, 299 (5607), 682.

(20) Holzmeister, P.; Acuna, G. P.; Grohmann, D.; Tinnefeld, P. Chem. Soc. Rev. 2014, 43 (4), 1014-1028.

(21) Punj, D.; Ghenuche, P.; Moparthi, S. B.; de Torres, J.; Grigoriev, V.; Rigneault, H.; Wenger, J. WIREs Nanomed. Nanobiotechnol. 2014, 6 (3), 268-282.

(22) Duan, H.; Hu, H.; Kumar, K.; Shen, Z.; Yang, J. K. W. ACS Nano 2011, 5 (9), 7593-7600.

(23) Kollmann, H.; Piao, X.; Esmann, M.; Becker, S. F.; Hou, D.; Huynh, C.; Kautschor, L.-O.; Bösker, G.; Vieker, H.; Beyer, A.; Gölzhäuser, A.; Park, N.; Vogelgesang, R.; Silies, M.; Lienau, C. Nano Lett. 2014, 14 (8), 4778-4784.

(24) Duan, H.; Hu, H.; Hui, H. K.; Shen, S.Z.; Yang, J. K. W Nanotechnology 2013, 24 (18), 185301.

(25) Wang, Y. M.; Lu, L.; Srinivasan, B. M.; Asbahi, M.; Zhang, Y. W.; Yang, J. K. W. Sci. Rep. 2015, 5, 9654.

(26) Flauraud, V.; van Zanten, T. S.; Mivelle, M.; Manzo, C.; Garcia Parajo, M. F.; Brugger, J. Nano Lett. 2015, 15 (6), 4176-4182.

(27) Thacker, V. V.; Herrmann, L. O.; Sigle, D. O.; Zhang, T.; Liedl, T.; Baumberg, J. J.; Keyser, U. F. Nat. Commun. 2014, 5, 3448.

(28) Kühler, P.; Roller, E.-M.; Schreiber, R.; Liedl, T.; Lohmüller, T.; Feldmann, J. Nano Lett. 2014, 14 (5), 2914-2919.

(29) Punj, D.; Regmi, R.; Devilez, A.; Plauchu, R.; Moparthi, S. B.; Stout, B.; Bonod, N.; Rigneault, H.; Wenger, J. ACS Photonics 2015, 2 (8), 1099-1107.

(30) Flauraud, V.; Mastrangeli, M.; Bernasconi, G. D.; Butet, J.; Alexander, D. T.; Shahrabi, E.; Martin, O. J.; Brugger, J. Nat. Nanotechnol. 2016, 12 (1), 73-80.

(31) Ghenuche, P.; Mivelle, M.; de Torres, J.; Moparthi, S. B.; Rigneault, H.; Van Hulst, N. F.; García-Parajó, M. F.; Wenger, J. Nano Lett. 2015, 15 (9), 6193-6201.

(32) Yang, J. K. W.; Cord, B.; Duan, H.; Berggren, K. K.; Klingfus, J.; Nam, S.-W.; Kim, K.-B.; Rooks, M. J. J. Vac. Sci. Technol. B 2009, 27 (6), 2622-2627.

(33) Nagpal, P.; Lindquist, N. C.; Oh, S.-H.; Norris, D. J. Science 2009, 325 (5940), 594-597.

(34) Zhou, W.; Odom, T. W. Nat. Nanotechnol. 2011, 6 (7), 423427.

(35) Aouani, H.; Wenger, J.; Gérard, D.; Rigneault, H.; Devaux, E.; Ebbesen, T. W.; Mahdavi, F.; Xu, T.; Blair, S. ACS Nano 2009, 3 (7), 2043-2048.

(36) Hohenester, U.; Ditlbacher, H.; Krenn, J. R. Phys. Rev. Lett. 2009, 103 (10), 106801.

(37) Langguth, L.; Femius Koenderink, A. Opt. Express 2014, 22 (13), 15397-15409.

(38) Khatua, S.; Yuan, H.; Orrit, M. Phys. Chem. Chem. Phys. 2015, 17 (33), 21127-21132.

(39) Kinkhabwala, A. A.; Yu, Z.; Fan, S.; Moerner, W. E. Chem. Phys. 2012, 406, 3-8.

(40) Kapusta, P. Absolute Diffusion Coefficients: Compilation of Reference Data for FCS Calibration. http://www.picoquant.com/ images/uploads/page/files/7353/appnote_diffusioncoefficients.pdf. (Accessed Jan 30, 2017).

(41) Bharadwaj, P.; Novotny, L. Opt. Express 2007, 15 (21), 1426614274.

(42) Aouani, H.; Mahboub, O.; Bonod, N.; Devaux, E.; Popov, E.; Rigneault, H.; Ebbesen, T. W.; Wenger, J. Nano Lett. 2011, 11 (2), $637-644$. 